\title{
Cancer Stem Cells Under Hypoxia as a Chemoresistance Factor in the Breast and Brain
}

\author{
Spencer W. Crowder • Daniel A. Balikov • \\ Yu-Shik Hwang $\cdot$ Hak-Joon Sung
}

Published online: 11 January 2014

(c) Springer Science+Business Media New York 2014

\begin{abstract}
Over the last 15 years, basic science and clinical studies have been conducted to identify cancer stem cells (CSCs) in several types of cancer to reveal their mechanistic involvement in cancer recurrence for therapeutic exploitation. Exposure of cancer cells and tissues to hypoxia, or sub-atmospheric concentrations of oxygen $\left(<21 \% \mathrm{O}_{2}\right)$, stimulates a variety of stress responses that bias the cells toward a self-preserving, anti-apoptotic phenotype, properties that are found in CSCs. Despite major advances in our understanding of hypoxia, CSCs, and their interrelated nature, some of the most promising cancer therapy has been of limited clinical efficacy, in part because of the inherently hypoxic nature of growing tumors. In this paper we discuss recent findings regarding the behavior of breast and brain CSCs under hypoxia, and the mechanisms of their chemo and/or radioresistance and metastatic potential.
\end{abstract}

Keywords Cancer stem cells - Hypoxia · Chemoresistance $\cdot$ Metastasis $\cdot$ Breast cancer . Brain cancer

S. W. Crowder · D. A. Balikov · H.-J. Sung $(\bowtie)$

Department of Biomedical Engineering, Vanderbilt University, VU Station B, Box 351631, Nashville, TN 37235, USA e-mail: hak-joon.sung@vanderbilt.edu

S. W. Crowder · D. A. Balikov · H.-J. Sung

Center for Stem Cell Biology, Vanderbilt University Medical Center, Nashville, TN, USA

Y.-S. Hwang · H.-J. Sung

Department of Maxillofacial Biomedical Engineering, School of Dentistry, Kyung Hee University, Seoul, South Korea

\section{Introduction}

Cancer stem cells (CSCs) are slow-dividing subpopulations of tumors with the ability to repopulate eradicated cancer cells by asymmetric cell division, resulting in cancer recurrence and patient mortality. CSCs have the same intrinsic properties as healthy stem cell populations found throughout the body, including the ability for self-renewal and capacity for multilineage differentiation [1]. Over the last 15 years, basic science and clinical studies have been conducted to identify CSCs in several types of cancer to reveal the complexities of their behavior for therapeutic exploitation.

Exposure of cells and tissues to hypoxia, or sub-atmospheric concentrations of oxygen $\left(<21 \% \mathrm{O}_{2}\right)$, stimulates a variety of stress response pathways that bias the cells toward a self-preserving, anti-apoptotic phenotype. As early as 1906, evidence for the protective effects of hypoxia against radiation, in both cancer and healthy tissue, began to accumulate in the literature [2,3]; however, dissection of hypoxia-dependent pathways has come to the forefront of cancer and radiation research only in the past few decades. Exposure of cells and tissues to hypoxia has revealed a tightly-regulated cascade of events that lead to increased expression of pro-survival factors that impart chemo and/or radioresistance [4-6]. In short, hypoxia stabilizes the hypoxia inducible factor- $\alpha$ proteins, which form a heterodimeric transcription factor complex. This complex binds the hypoxia-responsive elements of transcriptional targets involved in various pro-survival processes, for example angiogenesis, metabolism, and proliferation [7]. Because of this, the hypoxic state and the expression level of HIF1 $\alpha$ tend to correlate with more aggressive tumors and poor patient prognosis [8-10]. The exact mechanisms by which hypoxia induces its protective effects are 
complex and remain under investigation, but are broadly recognized to include:

- increased genomic instability and aberrant cell cycling $[8,11-16]$;

- dysregulated reactive oxygen species and redox mechanisms [17-22];

- a metabolic shift toward aerobic glycolysis [23-27]; and

- mitigated expression of pro-apoptotic factors [28-32].

Despite major advances in our understanding of hypoxia, CSCs, and their interrelated nature, some of the most promising cancer therapy has been of limited clinical efficacy over the past few years, in part because of the inherently hypoxic nature of growing tumors. In this paper we discuss recent findings regarding the behavior of breast and brain CSCs under hypoxia, and the mechanisms of their chemo and/or radioresistance and metastatic potential.

\section{Breast CSCs}

The involvement of CSCs in breast cancer was first discovered in 2003 when a CD $44^{+} \mathrm{CD} 24^{-/ \text {low }}$ Lineage $^{-}$subpopulation of tumor cells from human patient samples were shown to have tumor-initiating capacity in immunodeficient mice [33]. Interestingly, injection of as few as 100 of these tumorigenic cells gave rise to palpable tumors within 12 weeks. Continual serial passaging of these cells yielded a similar subpopulation. CD $44^{-}$cells from the patient tumor samples were non-tumorigenic, even when 10,000 cells were injected. In the years since, there has been an exponential increase in the number of breast cancer research studies attempting to identify the mechanisms by which breast CSCs resist classical chemotherapy and/or radiation and to identify the signaling that drives tumor recurrence.

Because tumors require blood perfusion to maintain viability and proliferation, anti-angiogenic therapy (i.e. therapy that prevents vascularization of the tumor) has been regarded as promising intervention for cancer treatment [34]. However, limited efficacy of anti-angiogenic therapy has been observed in clinical and pre-clinical studies, including studies of mouse models that demonstrated increased invasiveness and metastasis after inhibition of the vascular endothelial growth factor (VEGF) pathway [35, 36]. In 2009, Conley et al. [37] demonstrated that treatment with an anti-angiogenic agent, sunitinib, increased intratumoral hypoxia, which stimulated an increase in the number of aldehyde dehydrogenase (ALDH)-positive CSCs in vitro through a HIF1 $\alpha$-dependent mechanism. Furthermore, the tumors that developed after in vivo implantation of immortalized cancer cells were much larger in animals treated with sunitinib than in vehicle-treated controls.

Another recent study demonstrated an inverse correlation between the number of CSCs present in a given cell population and the enhanced chemoprotective effect of hypoxia in four immortalized cell lines and two samples derived from patients with recurring breast cancer [38]. Specifically, the resistance to radiation of cell populations in which the percentage of CSCs was low was greatly enhanced by culture under hypoxic conditions; however, for populations with a high percentage of CSCs, or for any cell population cultured in CSC-enriched mammospheres, no additional protective effect against radiation damage was observed when the cells were cultured under hypoxic, as compared with normoxic, conditions. The authors conducted gene-level screening of relevant antioxidative enzymes and concluded that expression of superoxide dismutase 2 (SOD2), but not SOD1, proportionally correlated with the number of breast CSCs in the population. These results suggest that breast CSCs have resistance to radiation via an SOD2-mediated, oxygen concentrationindependent mechanism.

The hypoxia-inducible protein carbonic anhydrase IX (CAIX) regulates tumor $\mathrm{pH}$ and cell survival by improving the transport of acids that accumulate within the tumor because of the large distances between cells and capillaries [39]. In a recent study, inhibition of CAIX gene expression or functional activity inhibited breast CSC expansion under hypoxia, an effect that was mediated downstream by the mammalian target of rapamycin complex 1 (mTORC1) [40]. Defined markers of tumorigenic breast CSCs $\left(\mathrm{CD} 44^{+} \mathrm{CD} 24^{- \text {low }}\right)$ were used to show that gene-level knockdown of CAIX reduced the number of breast CSCs in vitro, and their potential to undergo the epithelial-tomesenchymal transition (EMT). Furthermore, treatment with a specific, small-molecule inhibitor of CAIX, U-104, reduced the volume of orthotopic tumors in mice and virtually eliminated metastatic capability of primary tumors to the lungs, indicating the potential for CAIX as a novel therapeutic target for breast cancer treatment.

The status of estrogen receptor (ER) expression is also considered a strong indicator of the hypoxic response of breast CSCs, including mammosphere formation capacity and resistance to anti-angiogenic therapies [41 ${ }^{\bullet}$. In a recent article by Harrison et al. [41•], 13 patientderived samples and four immortalized cell lines were classified as either ER- $\alpha$-positive or negative, and their response to hypoxia was characterized. Mammosphere formation in vitro and tumor formation in vivo of ER- $\alpha$ positive cells, but not ER- $\alpha$-negative cells, was enhanced under $1 \%$ oxygen, as compared with $21 \%$ oxygen, through a hypoxia inducible factor 1-alpha (HIF1 $\alpha$ )dependent mechanism. Because Notch1 is a downstream 
mediator of ER- $\alpha$ and has been shown to be involved in breast CSC maintenance and proliferation [42], the authors further demonstrated that hypoxic culture of ER- $\alpha$-positive cells stimulated upregulation of Notch genes, and that mammosphere formation capacity under hypoxic conditions could be reduced by specifically blocking Notch activity with either gamma secretase inhibitor (GSI) or shRNA. Finally, the authors used an in-vivo xenograft model to demonstrate that the size of ER- $\alpha$-positive tumors correlated with the proportion of CSCs in the tumor, but an inverse correlation was observed for ER- $\alpha$-negative tumors. Taken together, this study reveals that ER status regulates the response to hypoxia in breast CSCs via Notch- and HIF1 $\alpha$-dependent pathways, and might provide new insights leading to anti-angiogenic clinical therapy.

To elucidate the specific involvement of Notch signaling in hypoxia-induced tumor metastasis, Xing et al. [43••] analyzed expression of Notch ligands in 779 breast cancer patients and identified a significant correlation between Jagged 2 expression and patient survival. Furthermore, Notch and Jagged 2 were strongly upregulated at the hypoxic invasive front in immunohistochemistry samples from 61 patients, and in-vitro hypoxic culture induced Jagged2 activation and EMT, an effect that was susceptible to blockage by the Notch inhibitor, GSI. This large-scale study confirmed involvement of the Notch pathway, specifically Jagged2, in hypoxia-driven breast cancer metastasis, and provided a potential prognostic marker for future clinical applications.

Another protein called CD44, a transmemebrane glycoprotein that binds hyaluronic acid, has been associated with aggressive, metastatic breast cancers [44]. A recent study investigated the relationship between hypoxia and a variety of CD44 isoforms in two immortalized breast cancer cell lines, MDA-MB-231 and SUM-149 [45]. Both cell lines were triple-negative (i.e. did not express estrogen receptor (ER), progesterone receptor (PR), or Her2-neu), indicative of the most aggressive, lethal forms of breast cancer. The authors induced hypoxia by exposure to either $0.2 \% \mathrm{O}_{2}$ or $200 \mu \mathrm{M} \mathrm{CoCl} \mathrm{Cl}_{2}$; this stimulated significant upregulation of two CD44 isoforms, and HIF1 $\alpha$ and HIF $2 \alpha$. By use of RNA interference techniques, hypoxiastimulated expression of CD44 variants 6 and 8 was shown to be regulated by HIF1 $\alpha$, but not HIF $2 \alpha$, at both the gene and protein-levels. Finally, CD44 expression was shown to correlate with regions of tumor hypoxia in vivo, confirming the in-vitro findings.

The tumor suppressor Period2 (PER2) is a circadian clock protein that, when lost, promotes invasion, metastasis, and the EMT, and correlates with poor prognosis in breast cancer patients through a mechanism of action that was recently described [46]. PER2 was shown to act as a transcriptional co-repressor by recruiting the polycomb proteins EZH2, SUZ12, and HDAC2 to the OCT4 binding sites of multiple EMT-related genes, including TWIST1 and $S L U G$, thereby preventing EMT and invasion. Furthermore, exposure to hypoxia resulted in degradation of PER2, which disrupted its repressive activity, and stimulated expression of EMT genes; this relationship was corroborated in clinical samples in which a negative correlation between hypoxia and PER2 was demonstrated.

With advances in general understanding of microRNAs (miRNAs), the involvement of a variety of these in regulating the response of breast CSCs to hypoxia is attracting interest. In 2011 Hwang-Verslues et al. [46] reported that miRNA-495 regulates the aggressiveness and hypoxic response of breast CSCs both in vitro and in vivo. When immortalized breast cancer cells devoid of CSCs were transfected with miRNA-495, significant increases in colony-formation ability and invasive capacity in vitro and tumor formation capacity (i.e. tumor volume) when implanted in vivo were observed. These results were shown to correlate with reduced E-cadherin and REDD1 expression. When cultured under low-oxygen conditions, miRNA-495 inhibited REDD1 expression, which promoted resistance to hypoxia, and stimulated an increase in the number of breast CSCs during the first two days.

Similar to the incorrect functionality of cell cycle regulators (e.g. p53) that leads to cancerous transformation and malignancy, dysregulation of oxygen-dependent stressresponse factors can impart detrimental characteristics to cancer cells. For example, aberrant activation of the hypoxia-responsive protein, HIF $2 \alpha$, under normoxic conditions has been shown to impart stem cell-like properties to immortalized cancer cells through an ALDH-dependent pathway [47]. Inhibition of ALDH reduced the proliferation and self-renewal of breast CSCs in vitro, and diminished tumor-initiating capacity and lung metastasis in vivo.

\section{Brain CSCs}

In addition to the wide-ranging involvement of CSCs in breast cancer, study of CSCs has also been fundamental to our understanding of the growth and metastatic potential of glioblastoma multiforme (GBM). Beginning in 2004, when CSCs in solid tumors were, primarily, known to exist in breast cancer only, two groups were able to separate and identify such CSCs from GBM tumors [48, 49]. Galli et al. [48], using isolated GBM patient lines, identified precursor cells with the functional capabilities of stem cells and determined that these stem cells had the capacity to form tumor masses. Similarly, Singh et al. [49] isolated GBM $\mathrm{CD}_{133^{+}}$cells with stem cell properties in vitro. When as few as 100 of these cells were implanted in NOD-SCID mouse brains, proliferative tumors were formed, whereas 
CD133- cells had the potential to engraft on to the brain only. Together, these studies formed the foundation for future work to study the involvement of CSCs in GBM and perhaps develop novel therapy targeting these cells.

In GBM, anti-VEGF treatment has not been effective because of evasive mechanisms. One study determined that GBM resistance was associated with upregulation of genes associated with mesenchymal origin and pro-inflammatory response factors [50]. Prolonged anti-VEGF therapy can also push GBM into mesenchymal and/or stem cell phenotypes that, over time, inhibit the effectiveness of the antiVEGF therapy [51]. Because of these observed responses, it is no surprise that induced hypoxic environments produce mesenchymal and stem cell phenotypes, as both cell populations are known to be located in low-oxygen environments. Hence, researchers have been focused on understanding the effect of hypoxia, specifically molecular mechanisms, in maintaining GBM CSCs and the resistance of GBM to anti-angiogenic therapy. On the basis of the pioneering studies in 2004, recent work has focused on further characterization of CSCs and their functional properties. One such characterization has been stemness markers and behavior. Li et al. [52] performed an in-vitro study to determine whether hypoxia enhanced stemness of CSCs. Hypoxia treatment of two established cell lines and primary glioma cells for $48 \mathrm{~h}$ promoted CD133 expression. OCT4 and SOX2 mRNA levels also increased significantly whereas GFAP (a marker of differentiation in neuronal cell lineages) decreased. When DAPT, an inhibitor of the Notch pathway, was added to the cells, the opposite trends were observed for OCT4, SOX2, and GFAP, leading the authors to speculate that hypoxia in some way dedifferentiates glioma cells into CSC-like populations. Similar trends were also seen in earlier work by a Danish group [53].

Although hypoxic environments have been proved to induce formation of stemness genes, more work has been conducted to study the behavior of crucial molecules that mediate the changes of stemness. Mathieu et al. [54••] took a broad approach by screening cell lines of different cancer types and human embryonic stem cells for overlapping changes of common genes when grown under hypoxic conditions. They showed that OCT4, NANOG, SOX2, KLF4, cMYC, and miRNA 302 were all induced under low oxygen tension in 11 cancer types, including that of the brain. Using OCT4 as a target gene of interest, the group found that when cancer cells were altered to express nondegradable HIF $1 \alpha$ and HIF $2 \alpha$ and OCT4-GFP, use of hypoxic culture conditions revealed a positive correlation between OCT4-GFP and non-degradable HIF levels. Subsequent experiments using siRNA to knockdown HIF1 $\alpha$ and HIF $2 \alpha$ showed the opposite trend, with reduced expression of hESC markers. The authors completed their findings by study of primary glioma-derived $\mathrm{CD} 133^{-}$cells, which they grew under hypoxic culture conditions. The low-oxygen conditions induced the cells to express the same hESC markers as before but also to form well-defined neurosphere structures associated with GBM. Thus, the authors suggested that HIF targets might act as crucial molecules affecting the dynamic states of stemness in cancer.

Although Mathieu et al. discovered that HIF1 $\alpha$ and HIF $2 \alpha$ were involved in the transformation of CSCs, their individual functions in the larger hypoxia signaling cascade had not yet been clarified. Bar et al. [55] contributed to this by determining whether HIF $1 \alpha$ or HIF $2 \alpha$ dominates long term phenotype characterization. Two glioma stem cell lines and three patient lines cultured under hypoxic conditions for $72 \mathrm{~h}$ had gene profiles that matched those in previous work by other groups, for example increases in CD133, HIF, KLF4, and SOX2. They also observed more prominent gene expression of LOX, HIG2, and VEGF and of downstream Notch pathway factor Hey2/Hes1 and ligands Jag1/Jag2. FACS confirmed that neurospheres formed under hypoxia comprised, primarily, $\mathrm{CD} 133^{+}$cells. Interestingly, if neurospheres with diameters as small as $100 \mu \mathrm{m}$ were seeded they would mature into larger neurospheres characteristic of previous in-vitro experiments whereas spheres with diameters less than $100 \mu \mathrm{m}$ did not mature. When mRNA and protein extraction was performed on these neurospheres, mRNA levels were higher for HIF1 $\alpha$ than HIF2 $\alpha$. In addition, only HIF1 $\alpha$ was expressed at the protein level. Bar et al. then studied xenograft models of the glioma stem cells (GSCs) and confirmed their in-vitro phenotype findings with one important caveat: hypoxia does not select for the best GSCs that maximize induced stemness transformation. HIF1 $\alpha$ may be of central importance in the transformation, but no physiological cutoffs indicating clonal selection from hypoxia were seen in the study.

In contrast with Bar et al., Seidel et al. [56] obtained results indicating that HIF $2 \alpha$ was the primary mediator of CSC transformation. Initial screening of candidate genes for isolating GSCs was based on three established glioblastoma cell lines grown under hypoxic conditions; this yielded 73 overlapping genes of interest, most of which were involved in the Wnt and TGF- $\beta$ pathways. Primary glioblastoma cell lines were then grown under $1 \%$ oxygen, and significant upregulation of CD133 and selected candidate genes ASPHD2, NFEL2L, MAML3, NFATc2, and ABL2 was observed. HIF also colocalized with these cells under hypoxia. By use of a Tet-off inducible system, the group determined that only HIF2 $\alpha$ led to a significant increase in expression of the candidate marker genes and 
that a major HIF2 $\alpha$ target was OCT4. Knockdown of HIF2 $\alpha$ confirmed its function as the primary mediator for glioblastoma tumor stem cell phenotype, because of reduced levels of CD133, ASPHD2, and MAML3. In contrast with reports claiming crucial involvement of HIF1 $\alpha$ in hypoxia-mediated CSC phenotypes, Seidel et al. found that HIF1 $\alpha$ reduced sphere formation. This observation had been made in other studies of HIF1 $\alpha$ [57].

Other research groups have also been interested in how hypoxia affects the ability of CSCs to form tumors. This has meant searching for less conventional downstream targets of HIF, and Heddleston et al. [58•] discovered histone methyl transferase mixed lineage leukemia 1 (MLL1) regulated tumorigenic potential in glioma stem cells. Xenograft-derived non-stem cells of glioblastoma were found to have increased levels of MLL1 under hypoxic conditions. When shRNA knockdown of HIF1 $\alpha$ and HIF2 $\alpha$ was introduced, MLL1 mRNA levels decreased, indicating MLL1 is a downstream target. When shRNA against MLL1 decreased HIF2 $\alpha$ expression and did not effect HIF1 $\alpha$ expression, ChIP analysis showed that HIF $2 \alpha$ bound to the MLL1 promoter and did not vary when grown under normoxic or hypoxic culture conditions. MLL1 specifically caused a loss of $\mathrm{H} 3 \mathrm{~K} 4 \mathrm{~m} 3$ and gain of $\mathrm{H} 3 \mathrm{~K} 27 \mathrm{~m} 3$, consistent with transcriptional activation of the HIF2 $\alpha$ locus. Other targets of MLL1 included PGK1 and VEGF, thus rationalizing MLL1's functional consequences of participating in some part in tumor angiogenesis. From a phenotypic perspective, loss of MLL1 greatly reduced cell proliferation and self-renewal, thus demonstrating its importance in GSC growth. This was further illustrated when shRNA-transfected glioblastoma cells against MLL1 greatly increased tumor latency in xenografts.

Finally, cancer stem cell researchers have focused on finding molecules responsible for the cells' interaction with or quasi-similarity to endothelial cell types involved in tumor vasculogenesis. As previously mentioned, tumors require a blood supply to sustain themselves and enable tumor growth, and it is known that GSCs expressing CDH5 can transdifferentiate into endothelial cells. Mao et al. [59] discovered that CDH5 mRNA levels were significantly higher in high-grade tumors than in low-grade tumors and that only tumor grade correlated with CDH5 levels. Compared with normal human brain, in which CDH5 is found on endothelial cells only, GBM samples also had GSCs expressing CDH5 and the proportion of GSCs with CDH5 increased with each passage (GSC5 at $8.4 \%$ and GSC11 at $11.5 \%$ ). Bioinformatic analysis of probable CDH5 interactions in these cells included HIF2 $\alpha$, VWF and PECAM-1, but HIF2 $\alpha$ was the most reliable predicted gene and known to be specifically expressed in endothelial cells. When cells were grown under hypoxic conditions, CDH5 mRNA increased 2.6 to 17.0-fold. shRNA knockdown of HIF $1 \alpha$ and HIF2 $\alpha$ also resulted in significant reduction in CDH5 in GSCs, and ChIP confirmed HIF $1 \alpha$ and HIF $2 \alpha$ interaction with the CDH5 promoter. Given these findings, the group knocked down CDH5 in GSCs grown in matrigel and found their capacity for tubulogenesis was severely diminished.

\section{Conclusion and Future Direction}

As revealed above, it is increasingly clear that hypoxic conditions stimulate CSCs to undergo metastatic transformation with improved survival by providing protective effects in both brain and breast tumor models. Continuous progress has been made in the elucidation of mechanisms driving the causative involvement of CSCs under hypoxia in cancer recurrence and failure of therapy. However, significant challenges still remain in attempts to understand the complex relationship among mechanistic factors within the context of spatiotemporal and patient-to-patient variations. Moreover, it is difficult to quantitatively investigate the effect of complex microenvironmental factors associated with hypoxia on CSC behavior by use of in-vivo models because of challenges associated with isolating specific microenvironmental interactions as well as discerning the effects of hypoxia on CSC-mediated cancer

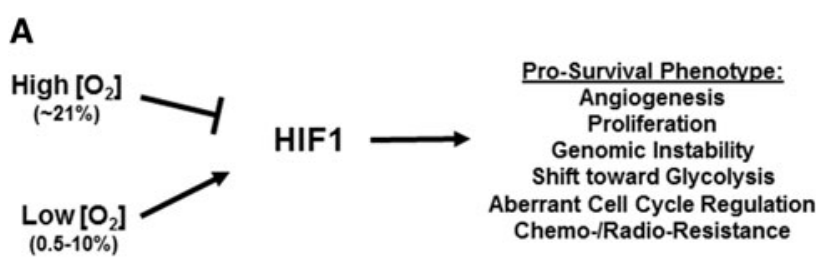

B

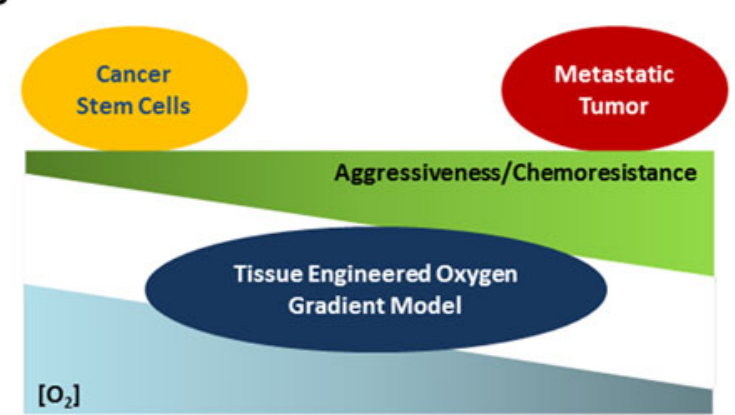

Fig. 1 a A general summary of the effects of hypoxia-induced HIF expression in CSCs. HIF activity is regulated by oxygen tension and promotes a pro-survival phenotype, including increases in angiogenesis, proliferation, genomic instability, and aberrant cell cycle regulation, and a shift toward glycolysis, all of which contribute to chemo and/or radioresistance. b A experimental model proposed for study of the effects on CSC behavior of different degrees of hypoxia, and how these effects can lead to metastatic potential and patient mortality 
recurrence and metastasis. One solution the authors suggest is to develop unique models that enable systematic investigation of hypoxic microenvironmental factors regulating CSC behavior and lead to discovery of therapeutic targets to prevent the contribution of CSCs to cancer recurrence and metastasis. For example, we have developed an environmentally relevant in-vitro model of carcinogenic hypoxia in which a metal carcinogen, nickel sulfate, is applied under hypoxic conditions [60]. By using this model we characterized the passage-dependent response of human stem cells in terms of early transformation in vitro, and the in-vivo consequences of implanted human stem cells. The results indicate that carcinogenic hypoxia modulates the activity of three critical transcription factors (c-MYC, p53, and HIF1), resulting in accumulation of reactive oxygen species and causing human stem cells to undergo cancerlike behavioral changes. Also, some promising 3D engineered tissue models have been developed by another group for study of cancer metastasis in vitro [61]. Moore et al. [62] developed an oxygen gradient model for study of vascular tissue remodeling. This type of tissue-engineering model could be used to investigate malignant transformation of CSCs under conditions of different oxygen content (Fig. 1). Considering the natural convergence of tissue engineering, stem cell biology, and cancer biology, collaborative efforts among researchers in these three fields seems a promising means of addressing the challenges.

\section{Compliance with Ethics Guidelines}

Conflict of Interest Spencer W. Crowder, Daniel A. Balikov, YuShik Hwang, and Hak-Joon Sung declare that they have no conflict of interest.

Human and Animal Rights and Informed Consent This article does not contain any studies with human or animal subjects performed by any of the authors.

\section{References}

Papers of particular interest, published recently, have been highlighted as:

- Of importance

-• Of major importance

1. Jordan CT, Guzman ML, Noble M (2006) Cancer stem cells. N Engl J Med 355(12):1253-1261. doi:10.1056/NEJMra061808

2. Schwarz G (1906) Ueber Desensibilisierung gegen Roentgen-und Radium-strahlen. Muenchner Medizinische Wochenschrift 56:1217-1218

3. Churchill-Davidson I, Sanger C, Thomlinson RH (1955) Highpressure oxygen and radiotherapy. Lancet 268(6874):1091-1095

4. Shrieve DC, Harris JW (1985) The in vitro sensitivity of chronically hypoxic EMT6/SF cells to X-radiation and hypoxic cell radiosensitizers. Int J Radiat Biol Relat Stud Phys Chem Med 48(1):127-138

5. Erler JT, Cawthorne CJ, Williams KJ, Koritzinsky M, Wouters BG, Wilson C et al (2004) Hypoxia-mediated down-regulation of Bid and Bax in tumors occurs via hypoxia-inducible factor 1-dependent and -independent mechanisms and contributes to drug resistance. Mol Cell Biol 24(7):2875-2889

6. Zolzer F, Streffer C (2002) Increased radiosensitivity with chronic hypoxia in four human tumor cell lines. Int $\mathrm{J}$ Radiat Oncol Biol Phys 54(3):910-920

7. Bertout JA, Patel SA, Simon MC (2008) The impact of $\mathrm{O}_{2}$ availability on human cancer. Nat Rev Cancer 8(12):967-975. doi: $10.1038 /$ nrc2540

8. Young SD, Marshall RS, Hill RP (1988) Hypoxia induces DNA overreplication and enhances metastatic potential of murine tumor cells. Proc Natl Acad Sci USA 85(24): 9533-9537

9. Zhang L, Hill RP (2004) Hypoxia enhances metastatic efficiency by up-regulating Mdm2 in KHT cells and increasing resistance to apoptosis. Cancer Res 64(12):4180-4189. doi:10.1158/00085472.CAN-03-3038

10. Zhang L, Hill RP (2007) Hypoxia enhances metastatic efficiency in HT1080 fibrosarcoma cells by increasing cell survival in lungs, not cell adhesion and invasion. Cancer Res 67(16):7789-7797. doi:10.1158/0008-5472.CAN-06-4221

11. Bristow RG, Hill RP (2008) Hypoxia and metabolism. Hypoxia, DNA repair and genetic instability. Nat Rev Cancer 8(3): 180-192. doi:10.1038/nrc2344

12. Unruh A, Ressel A, Mohamed HG, Johnson RS, Nadrowitz R, Richter E et al (2003) The hypoxia-inducible factor-1 alpha is a negative factor for tumor therapy. Oncogene 22(21):3213-3220. doi:10.1038/sj.onc.1206385

13. Hammer S, To KK, Yoo YG, Koshiji M, Huang LE (2007) Hypoxic suppression of the cell cycle gene CDC25A in tumor cells. Cell Cycle 6(15):1919-1926

14. Li CY, Little JB, Hu K, Zhang W, Zhang L, Dewhirst MW et al (2001) Persistent genetic instability in cancer cells induced by non-DNA-damaging stress exposures. Cancer Res 61(2):428-432

15. Reynolds TY, Rockwell S, Glazer PM (1996) Genetic instability induced by the tumor microenvironment. Cancer Res 56(24):5754-5757

16. Coquelle A, Toledo F, Stern S, Bieth A, Debatisse M (1998) A new role for hypoxia in tumor progression: induction of fragile site triggering genomic rearrangements and formation of complex DMs and HSRs. Mol Cell 2(2):259-265

17. Wang M, Kirk JS, Venkataraman S, Domann FE, Zhang HJ, Schafer FQ et al (2005) Manganese superoxide dismutase suppresses hypoxic induction of hypoxia-inducible factor-1 alpha and vascular endothelial growth factor. Oncogene 24(55): 8154-8166. doi:10.1038/sj.onc. 1208986

18. Dewhirst MW, Cao Y, Moeller B (2008) Cycling hypoxia and free radicals regulate angiogenesis and radiotherapy response. Nat Rev Cancer 8(6):425-437. doi:10.1038/nrc2397

19. Li F, Sonveaux P, Rabbani ZN, Liu S, Yan B, Huang Q et al (2007) Regulation of HIF-1alpha stability through S-nitrosylation. Mol Cell 26(1):63-74. doi:10.1016/j.molcel.2007.02.024

20. Quintero M, Brennan PA, Thomas GJ, Moncada S (2006) Nitric oxide is a factor in the stabilization of hypoxia-inducible factor1alpha in cancer: role of free radical formation. Cancer Res 66(2):770-774. doi:10.1158/0008-5472.CAN-05-0333

21. Moeller BJ, Cao Y, Li CY, Dewhirst MW (2004) Radiation activates HIF-1 to regulate vascular radiosensitivity in tumors: role of reoxygenation, free radicals, and stress granules. Cancer Cell 5(5):429-441

22. Nayak BK, Feliers D, Sudarshan S, Friedrichs WE, Day RT, New DD et al (2013) Stabilization of HIF-2alpha through redox 
regulation of mTORC2 activation and initiation of mRNA translation. Oncogene 32(26):3147-3155. doi:10.1038/onc.2012.333

23. Kim JW, Tchernyshyov I, Semenza GL, Dang CV (2006) HIF-1mediated expression of pyruvate dehydrogenase kinase: a metabolic switch required for cellular adaptation to hypoxia. Cell Metab 3(3):177-185. doi:10.1016/j.cmet.2006.02.002

24. Denko NC (2008) Hypoxia, HIF1 and glucose metabolism in the solid tumour. Nat Rev Cancer 8(9):705-713. doi:10.1038/nrc2468

25. Brahimi-Horn MC, Chiche J, Pouyssegur J (2007) Hypoxia signalling controls metabolic demand. Curr Opin Cell Biol 19(2):223-229. doi:10.1016/j.ceb.2007.02.003

26. Mathupala SP, Rempel A, Pedersen PL (2001) Glucose catabolism in cancer cells: identification and characterization of a marked activation response of the type II hexokinase gene to hypoxic conditions. J Biol Chem 276(46):43407-43412. doi:10. 1074/jbc.M108181200

27. Chen J, Zhao S, Nakada K, Kuge Y, Tamaki N, Okada F et al (2003) Dominant-negative hypoxia-inducible factor-1 alpha reduces tumorigenicity of pancreatic cancer cells through the suppression of glucose metabolism. Am J Pathol 162(4):1283-1291

28. Krieg M, Haas R, Brauch H, Acker T, Flamme I, Plate KH (2000) Up-regulation of hypoxia-inducible factors HIF-1alpha and HIF2alpha under normoxic conditions in renal carcinoma cells by von Hippel-Lindau tumor suppressor gene loss of function. Oncogene 19(48):5435-5443. doi:10.1038/sj.onc.1203938

29. Graeber TG, Osmanian C, Jacks T, Housman DE, Koch CJ, Lowe SW et al (1996) Hypoxia-mediated selection of cells with diminished apoptotic potential in solid tumours. Nature 379(6560): 88-91. doi:10.1038/379088a0

30. Gordan JD, Bertout JA, Hu CJ, Diehl JA, Simon MC (2007) HIF2alpha promotes hypoxic cell proliferation by enhancing c-myc transcriptional activity. Cancer Cell 11(4):335-347. doi:10.1016/ j.ccr.2007.02.006

31. Gordan JD, Lal P, Dondeti VR, Letrero R, Parekh KN, Oquendo $\mathrm{CE}$ et al (2008) HIF-alpha effects on c-Myc distinguish two subtypes of sporadic VHL-deficient clear cell renal carcinoma. Cancer Cell 14(6):435-446. doi:10.1016/j.ccr.2008.10.016

32. Liu MY, Poellinger L, Walker CL (2003) Up-regulation of hypoxia-inducible factor 2 alpha in renal cell carcinoma associated with loss of Tsc-2 tumor suppressor gene. Cancer Res 63(10): $2675-2680$

33. Al-Hajj M, Wicha MS, Benito-Hernandez A, Morrison SJ, Clarke MF (2003) Prospective identification of tumorigenic breast cancer cells. Proc Natl Acad Sci USA 100(7):3983-3988. doi:10. 1073/pnas.0530291100

34. Folkman J (1971) Tumor angiogenesis: therapeutic implications. N Engl J Med 285(21):1182-1186. doi:10.1056/NEJM1971111 82852108

35. Ebos JM, Lee CR, Cruz-Munoz W, Bjarnason GA, Christensen JG, Kerbel RS (2009) Accelerated metastasis after short-term treatment with a potent inhibitor of tumor angiogenesis. Cancer Cell 15(3):232-239. doi:10.1016/j.ccr.2009.01.021

36. Paez-Ribes M, Allen E, Hudock J, Takeda T, Okuyama H, Vinals $\mathrm{F}$ et al (2009) Antiangiogenic therapy elicits malignant progression of tumors to increased local invasion and distant metastasis. Cancer Cell 15(3):220-231. doi:10.1016/j.ccr.2009.01.027

37. Conley SJ, Gheordunescu E, Kakarala P, Newman B, Korkaya H, Korkaya H, Heath AN et al (2012) Antiangiogenic agents increase breast cancer stem cells via the generation of tumor hypoxia. Proc Natl Acad Sci USA 109(8):2784-2789. doi:10. 1073/pnas.1018866109

38. Lagadec C, Dekmezian C, Bauche L, Pajonk F (2012) Oxygen levels do not determine radiation survival of breast cancer stem cells. PLoS One 7(3):e34545. doi:10.1371/journal.pone.0034545

39. Swietach P, Hulikova A, Vaughan-Jones RD, Harris AL (2010) New insights into the physiological role of carbonic anhydrase IX in tumour pH regulation. Oncogene 29(50):6509-6521. doi:10. 1038/onc.2010.455

40. Lock FE, McDonald PC, Lou Y, Serrano I, Chafe SC, Chafe SC, Ostlund $\mathrm{C}$ et al (2013) Targeting carbonic anhydrase IX depletes breast cancer stem cells within the hypoxic niche. Oncogene. doi:10.1038/onc.2012.550

41. - Harrison H, Rogerson L, Gregson HJ, Brennan KR, Clarke RB, Landberg G (2013) Contrasting hypoxic effects on breast cancer stem cell hierarchy is dependent on ER-alpha status. Cancer Res 73(4):1420-1433. doi: 10.1158/0008-5472.CAN-12-2505. Using immortalized cell lines and patient tissue samples, this paper demonstrated contrasting effects of hypoxia on CSC enrichment, based upon estrogen receptor-alpha status of the overall tumor

42. Harrison H, Farnie G, Howell SJ, Rock RE, Stylianou S, Brennan KR et al (2010) Regulation of breast cancer stem cell activity by signaling through the Notch4 receptor. Cancer Res 70(2):709-718. doi:10.1158/0008-5472.CAN-09-1681

43. •• Xing F, Okuda H, Watabe M, Kobayashi A, Pai SK, Liu W et al (2011) Hypoxia-induced Jagged2 promotes breast cancer metastasis and self-renewal of cancer stem-like cells. Oncogene 30(39):4075-4086. doi:10.1038/onc.2011.122. Investigation of Notch ligand expression from $>700$ patients in GEO database and $>60$ clinical samples showed that Jagged 2 and Notch expression correlate negatively with positive clinical outcome and metastasis-free survival. This study provides a potential prognostic indicator for breast cancer diagnostics

44. Peng ST, Su CH, Kuo CC, Shaw CF, Wang HS (2007) CD44 crosslinking-mediated matrix metalloproteinase-9 relocation in breast tumor cells leads to enhanced metastasis. Int $\mathrm{J}$ Oncol 31(5):1119-1126

45. Krishnamachary B, Penet MF, Nimmagadda S, Mironchik Y, Raman V, Solaiyappan M et al (2012) Hypoxia regulates CD44 and its variant isoforms through HIF-1alpha in triple negative breast cancer. PLoS One 7(8):e44078. doi:10.1371/journal.pone. 0044078

46. Hwang-Verslues WW, Chang PH, Wei PC, Yang CY, Huang CK, Kuo WH et al (2011) miR-495 is upregulated by E12/E47 in breast cancer stem cells, and promotes oncogenesis and hypoxia resistance via downregulation of E-cadherin and REDD1. Oncogene 30(21):2463-2474. doi:10.1038/onc.2010.618

47. Kim RJ, Park JR, Roh KJ, Choi AR, Kim SR, Kim PH et al (2013) High aldehyde dehydrogenase activity enhances stem cell features in breast cancer cells by activating hypoxia-inducible factor-2alpha. Cancer Lett 333(1):18-31. doi:10.1016/j.canlet. 2012.11.026

48. Galli R, Binda E, Orfanelli U, Cipelletti B, Gritti A, De Vitis S et al (2004) Isolation and characterization of tumorigenic, stemlike neural precursors from human glioblastoma. Cancer Res 64(19):7011-7021. doi:10.1158/0008-5472.CAN-04-1364

49. Singh SK, Hawkins C, Clarke ID, Squire JA, Bayani J, Hide T et al (2004) Identification of human brain tumour initiating cells. Nature 432(7015):396-401. doi:10.1038/nature03128

50. Piao Y, Liang J, Holmes L, Henry V, Sulman E, de Groot JF (2013) Acquired resistance to anti-VEGF therapy in glioblastoma is associated with a mesenchymal transition. Clin Cancer Res 19(16):4392-4403. doi:10.1158/1078-0432.CCR-12-1557

51. Piao Y, Liang J, Holmes L, Zurita AJ, Henry V, Heymach JV et al (2012) Glioblastoma resistance to anti-VEGF therapy is associated with myeloid cell infiltration, stem cell accumulation, and a mesenchymal phenotype. Neurooncology 14(11):1379-1392. doi:10. 1093/neuonc/nos158

52. Li P, Zhou C, Xu L, Xiao H (2013) Hypoxia enhances stemness of cancer stem cells in glioblastoma: an in vitro study. Int J Med Sci 10(4):399-407. doi:10.7150/ijms.5407

53. Kolenda J, Jensen SS, Aaberg-Jessen C, Christensen K, Andersen $\mathrm{C}$, Brunner $\mathrm{N}$ et al (2011) Effects of hypoxia on expression of a 
panel of stem cell and chemoresistance markers in glioblastomaderived spheroids. J Neurooncol 103(1):43-58. doi:10.1007/ s11060-010-0357-8

54. •• Mathieu J, Zhang Z, Zhou W, Wang AJ, Heddleston JM, Pinna $\mathrm{CM}$ et al (2011) HIF induces human embryonic stem cell markers in cancer cells. Cancer Res 71(13):4640-4652. doi:10.1158/00085472.CAN-10-3320. Compared various cancer lines lines to human embryonic stem cells grown under hypoxia to located overlapping gene expression profiles. This study discovered that there were trends in stemness markers and hypoxia inducible factors

55. Bar EE, Lin A, Mahairaki V, Matsui W, Eberhart CG (2010) Hypoxia increases the expression of stem-cell markers and promotes clonogenicity in glioblastoma neurospheres. Am J Pathol 177(3):1491-1502. doi:10.2353/ajpath.2010.091021

56. Seidel S, Garvalov BK, Wirta V, von Stechow L, Schanzer A, Meletis K et al (2010) A hypoxic niche regulates glioblastoma stem cells through hypoxia inducible factor 2 alpha. Brain 133(Pt 4):983-995. doi:10.1093/brain/awq042

57. Mendez O, Zavadil J, Esencay M, Lukyanov Y, Santovasi D, Wang SC et al (2010) Knock down of HIF-1alpha in glioma cells reduces migration in vitro and invasion in vivo and impairs their ability to form tumor spheres. Mol Cancer 9:133. doi:10.1186/ 1476-4598-9-133

58. - Heddleston JM, Wu Q, Rivera M, Minhas S, Lathia JD, Sloan $\mathrm{AE}$ et al (2012) Hypoxia-induced mixed-lineage leukemia 1 regulates glioma stem cell tumorigenic potential. Cell death and differentiation 19(3):428-439. doi:10.1038/cdd.2011.109. Determined that MLL1 was a downstream target of hypoxia inducible factor that altered methylation profiles of glioblastoma stem cells. This paper was one of the first to show epigenetic changes as a consequence of hypoxia inducible factor expression from low oxygen settings

59. Mao XG, Xue XY, Wang L, Zhang X, Yan M, Tu YY et al (2013) CDH5 is specifically activated in glioblastoma stemlike cells and contributes to vasculogenic mimicry induced by hypoxia. Neurooncology 15(7):865-879. doi:10.1093/neuonc/ not029

60. Crowder SW, Horton LW, Lee SH, McClain CM, Hawkins OE, Palmer AM et al (2013) Passage-dependent cancerous transformation of human mesenchymal stem cells under carcinogenic hypoxia. Faseb J 27(7):2788-2798. doi:10.1096/fj.13-228288

61. Szot CS, Buchanan CF, Freeman JW, Rylander MN (2011) 3D in vitro bioengineered tumors based on collagen I hydrogels. Biomaterials 32(31):7905-7912. doi:10.1016/j.biomaterials. 2011.07.001

62. Moore M, Moore R, McFetridge PS (2013) Directed oxygen gradients initiate a robust early remodeling response in engineered vascular grafts. Tissue Eng Part A 19(17-18):2005-2013. doi:10.1089/ten.TEA.2012.0592 